\title{
Trend of Malaria Cases in Hana and Keyafer Health Centers, South Omo Zone, Southern Ethiopia
}

\author{
Solomon Ergete ${ }^{1}$, Solomon Sorsa ${ }^{2}$, Eskindir Loha ${ }^{3}$, Solomon Asnake ${ }^{3 *}$
}

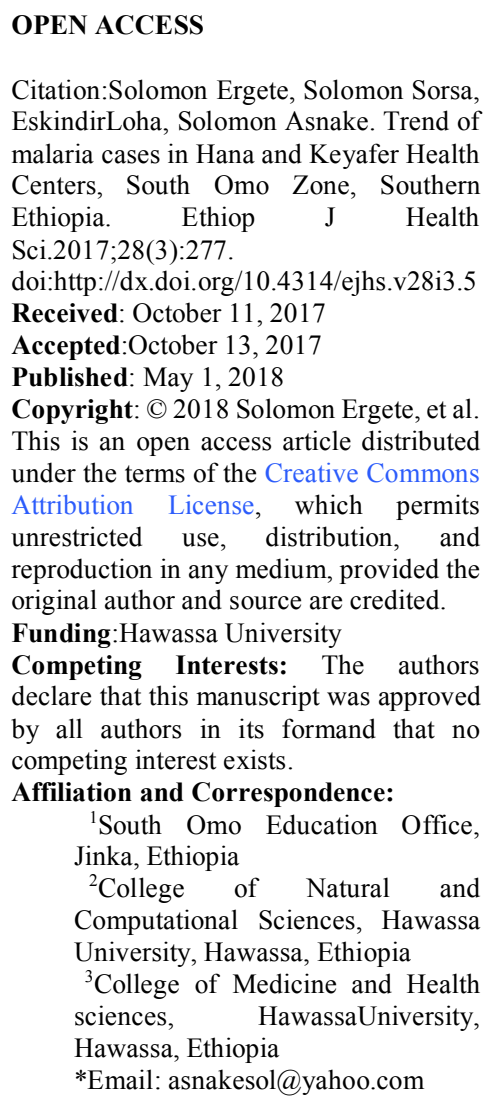

OPEN ACCESS

Citation:Solomon Ergete, Solomon Sorsa, EskindirLoha, Solomon Asnake. Trend of malaria cases in Hana and Keyafer Health Centers, South Omo Zone, Southern Ethiopia. Ethiop J Health Sci.2017;28(3):277.

doi:http://dx.doi.org/10.4314/ejhs.v28i3.5 Received: October 11, 2017

Accepted:October 13, 2017

Published: May 1, 2018

Copyright: (C) 2018 Solomon Ergete, et al.

This is an open access article distributed under the terms of the Creative Commons Attribution License, which permits unrestricted use, distribution, and reproduction in any medium, provided the original author and source are credited.

Funding:Hawassa University

Competing Interests: The authors declare that this manuscript was approved by all authors in its formand that no competing interest exists.

Affiliation and Correspondence:

${ }^{1}$ South Omo Education Office, Jinka, Ethiopia

${ }^{2}$ College of Natural and Computational Sciences, Hawassa University, Hawassa, Ethiopia ${ }^{3}$ College of Medicine and Health sciences, HawassaUniversity, Hawassa, Ethiopia

*Email: asnakesol@yahoo.com

\begin{abstract}
BACKGROUND: Data registered in health facilities about malaria are vital source of information regarding the trend and pattern of the disease. Moreover the data also used to evaluate the impact of the intervention methods and to plan malaria control accordingly. The aim of this study was to assess the number of malaria cases in the service providing health institutions of South Omo Zone, SNNPR, over a period of seven years, 2008-2014.

METHODS: A retrospective review of routinely collected and recorded data on malaria was conducted at Hana and Keyafer health centers located in South OmoZone, during December-March/2014.

RESULT: This retrospective study examined records of 54,160 presumptive and confirmed malaria cases registered over seven years (January 2008-December 2014). Among the registered, a total of 22,494 laboratory confirmed malaria cases were reported. The mean annual prevalence of malaria was 3213, with total slide positivity rate (SPR) of $41.53 \%$. The seven years'overall trend of malaria occurrence in the study sites showed a fluctuating trend from 2008 to 20114.P. falciparum was the predominant species with no trend shift in seven years period. Plasmodium falciparum accounted for $61.03 \%$ and Plasmodium vivax for $32.44 \%$. Most of the confirmed cases were males (63.26\%) and most were above 15 years old (70.7\%). Rainfall at lag of one month was significantly predicting number of cases at Hana Health center $(P<0.001)$, though it was found to be not significant in case of KeyaferHealth Center.

CONCLUSION: The finding of the retrospective study revealed that malaria still remains as a public health problem in the study areas. The deadly species $P$. falciparium was most predominant, and the age group above 15 was more infected. Hence, malaria intervention methods should be scaled up in the study sites.

KEYWORDS: Malaria, cases, health institution
\end{abstract}

\section{INTRODUCTION}


Malaria is a life-threatening infectious disease caused by the protozoan parasite, Plasmodium falciparum (P. falciparum), P. malariae, P. ovale, $P$. vivax and $P$. knowlesi(1). Despite currentlyavailable interventions, malaria remains a major public health problem. There were estimated212 million cases of malaria and 429,000 deaths worldwide in 2015(2). More than $80 \%$ of the cases and $90 \%$ of all deaths occur in sub-Saharan Africa, with $77 \%$ occurring in childrenunder five years of age (3). Malaria also remains a major global development challenge; inAfrica it accounts for $40 \%$ of public health expenditure and $25 \%$ of the household income (4).Malaria is the leading cause of morbidity and mortality in Ethiopia; approximately $75 \%$ oflandmass is endemic for malaria and about $68 \%$ of the total population living in areas at risk ofmalaria (5).Approximately, $80 \%$ of the 736 districts in Ethiopia are considered malarious (6).According to the $\mathrm{FMoH} \mathrm{(7),}$ approximately, 3.4 million cases of malaria (both laboratoryconfirmed and clinically diagnosed) were reported. Among the four plasmodium species knownto cause malaria in Ethiopia, the two epidemiologically important species are $P$. falciparum and $P$. vivax comprising $60 \%$ and $40 \%$ respectively (7). As reported by the Ethiopia's FMoH (8), in 2009, malaria was the first cause of outpatient visits, health facility admissions and $29 \%$ inpatient deaths, accounting for $12 \%$ of outpatient visits and $9.9 \%$ of admissions. In the Southern Nations,Nationalities and Peoples' Region (SNNPR), about $65 \%$ of the population is living in malariaendemic areas, and malaria is the primary cause of outpatient and inpatient consultations andhospital deaths in the region (9).Studies indicated that after the introduction of the current malaria control strategies, morbidity and mortality due to malaria are decreasing in the country, though malaria still remains a major health problem (10). Analysis of data relevant to malaria from healthcare centers and health facility records provides essential information needed to evaluate the national malaria control program and gives insight to the changing malaria situation, which intern guides modification of malaria control program $(11,12)$. The trend of malaria episode in the study areaswas not determined. Hence, this study was intended to determine the last seven years' (2008-2014)trend of malaria occurrence in South Omo Zone, Southern Ethiopia.

\section{MATERIALS AND METHODS}

The study areas: The study was conducted in Hana and Keyafer health centers found in SalamagoandBenatsemay districts of South OmoZone. The two districts are situated at 5.320 6. 460 Northand 35. 890-36.450 East and 5.110 5.700 North and 36.200-37.040 respectively. The districts are located about $42 \mathrm{~km}$ from the zonal capital, Jinka and $483 \mathrm{~km}$ from Hawassa, the regional capital. The districts lie on an average altitude of 1500 meter above sea level and ranges from 500 to 2500 masl (13). Annual rainfall ranges between $400-1600 \mathrm{~mm}$, and the main rainy seasons are March, April and July while the light rainy seasons are August and September. The two districts have 3 health centers each and 18 and 31 health posts respectively. The total population size of Salamago was 33,066 of whom 16,717 were males and 16349 females, whereas that of Benatsemay was 62,825 of which 31788 were males and 31037 females (14). Among the health centers in the zone, Hana from Salamago and Keyafer from Benatsemay are the oldest and relatively better in health facility, health professional staff and document keeping techniques.

Study design and study population:A health facility based retrospective cross-sectional study was conducted at Hana and Keyafer health centersbetween $2008-2014$. The study included all records of individuals registered on $\log$ books from 2008 to 2014 and diagnosed for malaria both clinically and via blood film test.

Sampling technique and procedures:The districts and health centers were selected purposively based on the high rates of malaria case burden relative to other districts in the zone. Data was collected from institutional registration books of the two health centers by health personnel on prepared format sheets. Individual leveldata on malaria diagnosis results, type of species, dates of diagnoses and available demographicdata were registered.

DOI: http://dx.doi.org/10.4314/ejhs.v28i3.5 
Moreover, using a questionnaire,malaria intervention activities and other required information were collected from the respective health centers and districts health offices. The meteorological data of the study areas (annual, monthly, maximum and minimum rain fall and temperature) were collected from Regional Meteorology Agency.

Data quality management:The study used confirmed cases that were registered on the national malaria registration book of the two health facilities. A one-day training was given for data collectors on data extraction.

Data analysis: The data on malaria case records of the study sites were transferred to Excel spreadsheet and checked for completeness. The data were exported and analyzed using SPSS statistical software version 20 for windows. For the sake of comparison of burden of malaria in the two set-ups, the total catchment population was used as a denominator to come up with the number of cases per 1000 population. Linear regression analysis was used to estimate the level of differences in the trends of malaria cases between the study areas and to determine the significance level among different variables. Cross-correlations were done between malaria cases and meteorological factors; the significant pick was observed at lag number of -1 for malaria cases and corresponding rainfall data. Linear regression model was built using rainfall data at lag of onemonth to predict malaria cases of the subsequent month.

Ethical considerations:The proposal was review by the Review Board of the Department of Biology and approved by the Research and Technology Transfer Directorate Office of Hawassa University. The purpose of the study was explained to the concerned bodies in Hana and Keyafer health centers, and permission was obtained from the two health centers and their respective district health offices.

\section{RESULTS}

Over seven years (January 2008-December 2014), 54,160 presumptive and confirmed malaria cases wereexamined. Over two-third of the cases were from Hana health Center, and close to one-third of the cases were from Keyafer. A total of 22,494 laboratory confirmed malaria cases were reported, and the mean annual prevalence of malaria was 3213 , with total slide positivity rate (SPR) of $41.53 \%$. The seven years' trend of malaria occurrence showed a fluctuating trend. There was irregularity on the number of malaria cases, with the minimum (831) microscopically confirmed cases reported in 2009, and the maximum (5648) confirmed cases were reported in 2012 (Figure 1). The prevalence had shown slight fall from 2008 to 2009 . Then, from 2010 to 2012 , it had shown a sharp rise, and peak malaria case was reported in 2012 after which the prevalence had shown a sharp fall till 2014. Generally, as the finding indicated, no significant reduction in laboratory confirmed malaria cases was recorded during the seven years' period.

Both $P$. falciparum and $P$. vivax were reported each year, and both showed year-to-year increment till 2012 then fell down. In all considered years $P$. falciparum was the predominant species with no trend shift. $P$. falciparum accounts for $13727(61.03 \%)$ cases, while $P$. vivax accounts for $7297(32.44 \%)$ and $1470(6.53 \%)$ were recorded as mixed. The prevalence of $P$. falciparumremainedconsistent from 2008-2009 and then showed steady increment till 2012 where it reached climax and dropped down sharply in 2013 and 2014. P. vivax cases kept constant till 2010 and then increased gradually up to 2012 and fell gradually in 2013 and 2014. The number of mixed infection cases almost kept constant except minor peak seen in 2012 (Figure 2). 


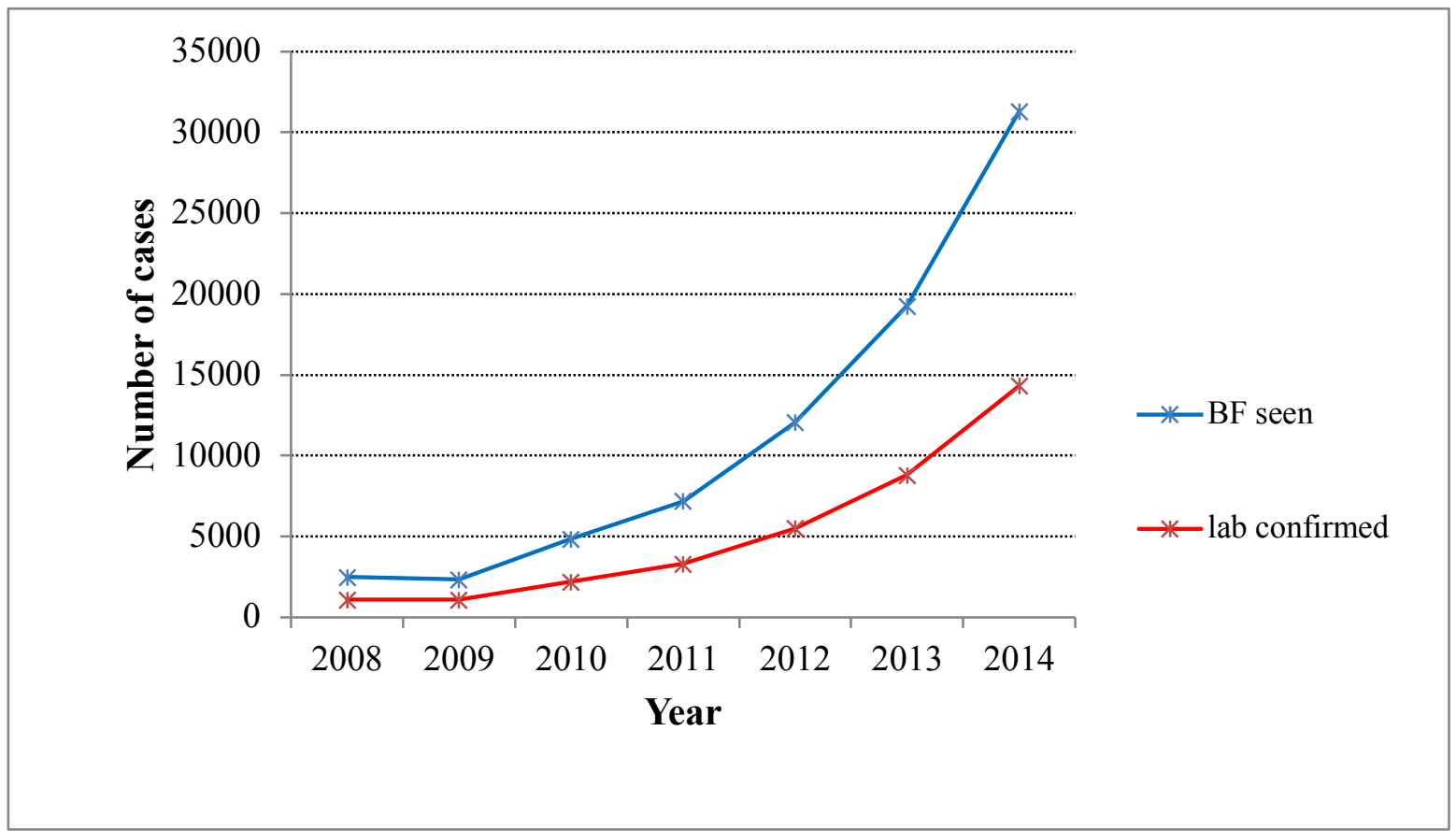

Figure 1:Seven years' trend of laboratory confirmed malaria cases, South Omo Zone, SNNPR, from January 2008 - December 2014.

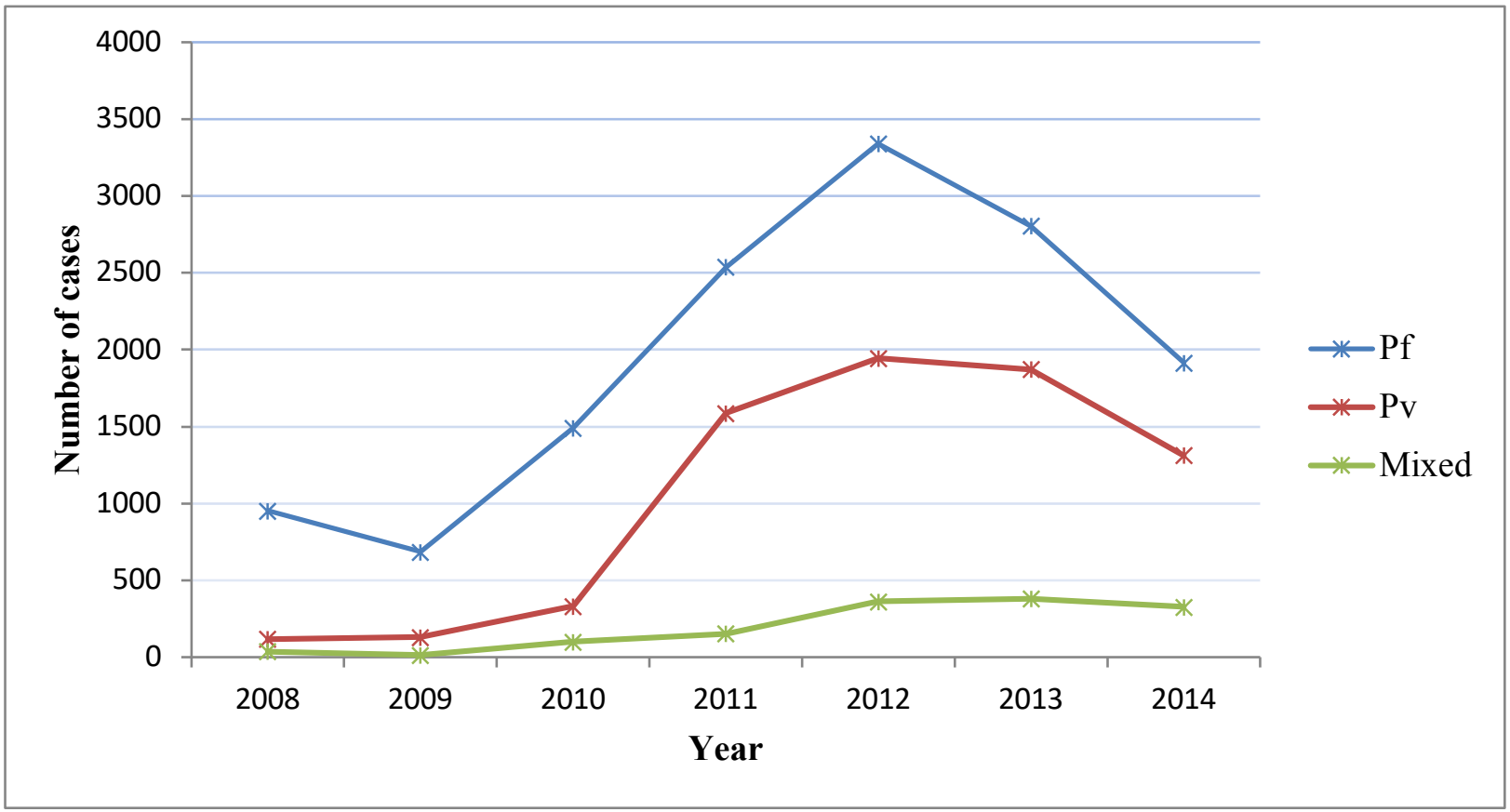

Figure 2: Trend of confirmed malaria cases by species composition and year, South Omo Zone, SNNPR, from January 2008 - December 2014

DOI: http://dx.doi.org/10.4314/ejhs.v28i3.5 
Regarding the overall proportion of sex, $61 \%$ of the total patients examined were males while $39 \%$ were females. Of the total malaria slide-positives, microscopically confirmed that male malaria cases were 14229 (63.26\%), while females were 8265 (36.74\%). The data (Table 1) shows that overall, more males were examined than females and consequently more males were found infected. However, although malaria prevalence is higher among males, from 2010 up to 2014 in 2008, it was nearly equal and even in 2009 the prevalence was slightly less than females.

Table 1: Slide positive rate of malaria cases by sex and year, South Omo Zone, SNNPR, from January 2008 - December 2014

\begin{tabular}{llllll}
\hline \multirow{2}{*}{ Year } & Total cases & \multicolumn{3}{c}{ Malaria positive cases } & Percentage \\
\cline { 3 - 5 } & screened & Male & Female & Total & $(\%)$ \\
\hline 2008 & 2918 & 568 & 534 & 1102 & 37.76 \\
2009 & 2351 & 398 & 433 & 831 & 35.35 \\
2010 & 4987 & 1141 & 781 & 1922 & 38.54 \\
2011 & 9126 & 2428 & 1850 & 4278 & 46.87 \\
2012 & 12287 & 3844 & 1804 & 5648 & 45.96 \\
2013 & 12195 & 3345 & 1713 & 5058 & 41.46 \\
2014 & 10296 & 2505 & 1150 & 3655 & 35.49 \\
Total & 54160 & 14229 & 8265 & 22494 & 41.53 \\
\hline
\end{tabular}

There was variation of trend of confirmed malaria cases among different age groups as well as year-to-year trends. As indicated in Figure 3, malaria was reported in all age groups, but the age group of above 15 years was more affectedthan the other age groups. The prevalence of malaria was highest in this age category from 2008 to 2014, and it reached climax in 2012. In general, the overall proportion of cases in the age group of above 15 years was $68.90 \%$, followed by $5-14$ year old $(15.42 \%)$ then by $1-4$ year old $(10.88 \%)$ and $(3.8 \%)$ in less than 1 year old (Figure 3). Although individuals in age group of 5-14 years were the second most affected groups followed by age groups of 1-4. There was no much pronounced difference in the number of cases between the two age groups. On the other hand, less than one year age category was the least affected; in this group the prevalence of malaria kept stable except the minor peak seen in 2010. $P$. falciparum was exclusively the dominant species in all age groups, and it was higher in the above 15 year olds followed by 5-14 year olds, 1-4 year olds and less than 1 year olds. Although mixed infections were insignificant among all age group segments, they were more relatively prevalent among the above 15 years age group. The chisquare test also showed presence of positive association between age and malaria cases (X2 = 127; $\mathrm{P}<0.001)$. 


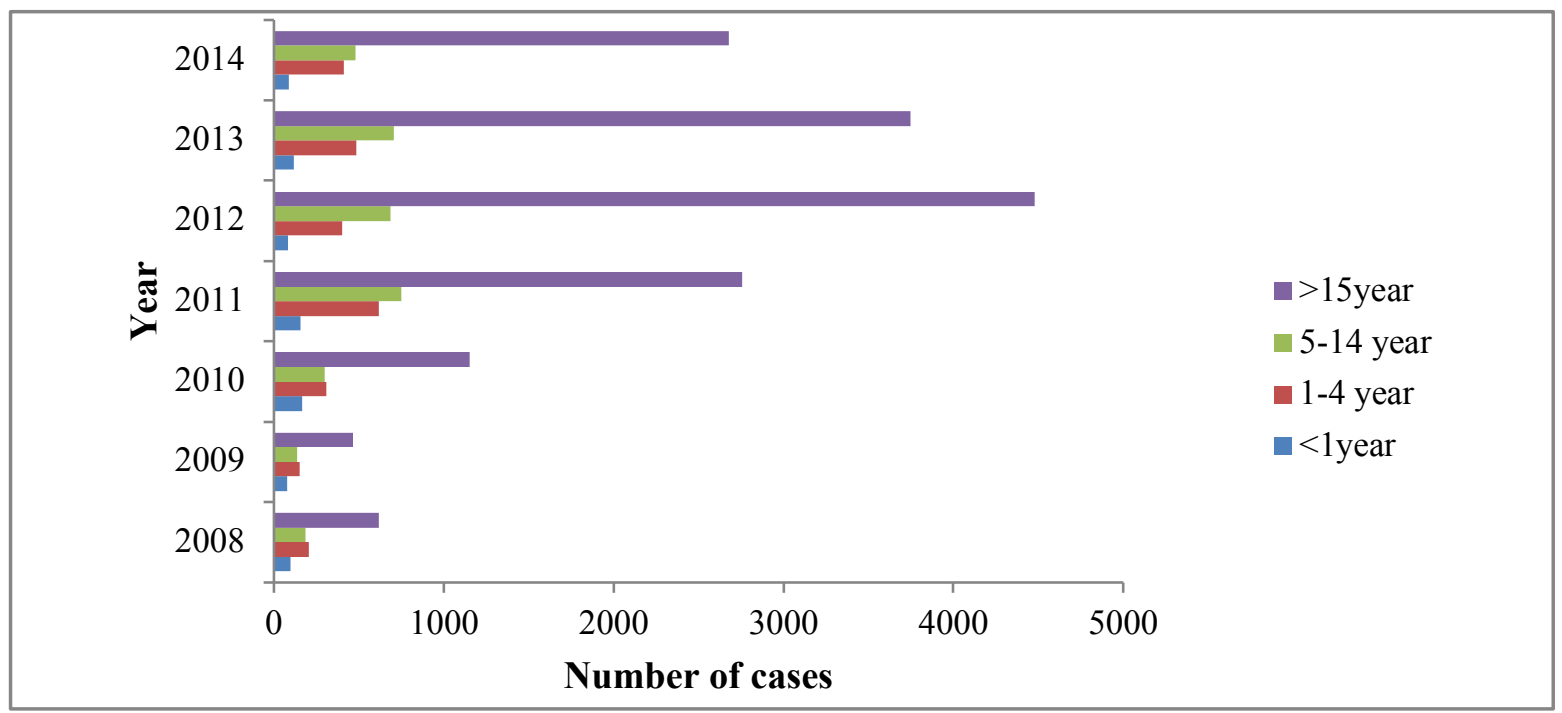

Figure 3:Trend of confirmed malaria cases by age group and year, South Omo Zone, SNNPR, from January 2008 - December 2014.

Although malaria occurred in all months, the average monthly distribution of malaria prevalence exhibited different patterns during the seven years' period (Figure 4). Overall, two major peaks were noticed; the first peaks occurred from September up to November (spring), and the second peak occurred between April and June (autumn through summer). The highest peak of malaria cases were observed during autumn through summer, and the lowest number of malaria cases were observed during winter season (January, February and March). As compared to $P$. vivax thecontribution of P. falciparum was almost double throughout the seasons from 2008-2014.

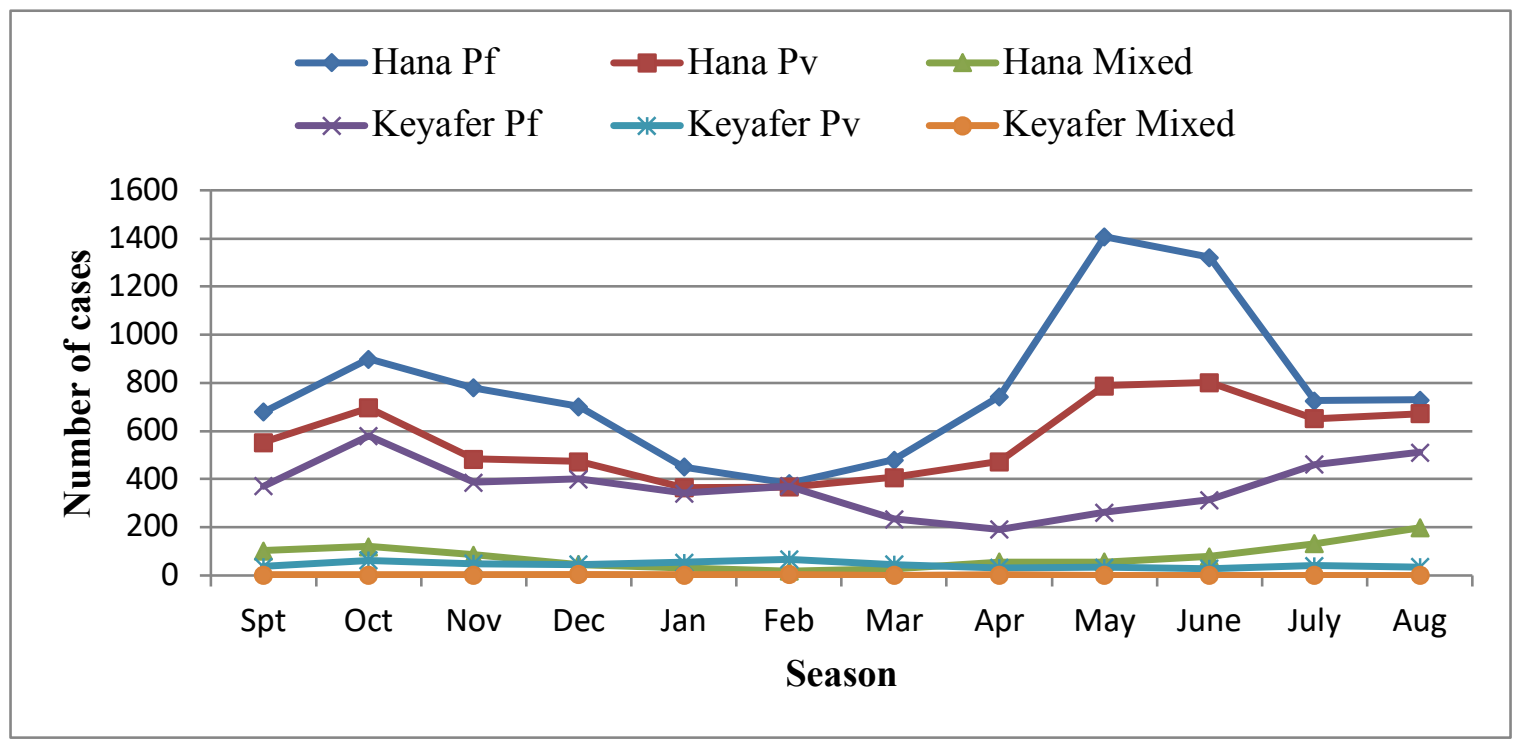

Figure 4: Trend of confirmed malaria case by study sites, South Omo Zone, SNNPR,from January 2008 December 2014

DOI: http://dx.doi.org/10.4314/ejhs.v28i3.5 
There was also a difference in the pattern of malaria prevalence between the study sites. Generally, the seven years' data analyses indicated that above twothird $(71 \%)$ of the cases were from Hana Health Center and close to one-third (29\%) of the cases were from KeyaferHealth Center. During theseven years' period considered, the number of cases increased steadily from 2008-2012 in Hana Health Center and exhibited two major peak in 2012 and dropped sharply in 2013 and 2014. On the other hand, the prevalence of malaria in KeyaferHealth Center was much lower than that of Hana Health Center. In this site, during the seven years' period, relatively stable prevalence was observed except the minor peak observed in 2011.

Cross-correlation between malaria cases and meteorological factors indicated that the majorsignificantpeak was observed at lag number of -1 for malaria cases of Hana Health Center and the corresponding rain fall data. The linear regression models built by rainfall data at lag of one month were capable of explaining the total variations in the number of falciparum and vivax cases by $25.5 \%$ (R2 $=0.255)$ and $22.5 \%(\mathrm{R} 2=0.225)$, respectively. The data showed that rainfall at lag of one month was significantly predicted the number of $P$. falciparum cases and $P$. vivax cases of Hana Health Center ( $\mathrm{P}$ $<0.001)$. Neither minimum nor maximum temperature be significantly predicted the number of malaria cases. Meanwhile, none of the lags of meteorological variables was found to be significant for KeyaferHealth Center.

\section{DISCUSSION}

This healthcare service based retrospective study assessed seven years' period distribution of malaria among individuals by place, time and composition of Plasmodium species based on the available data of two health service institutions. As the finding indicated, the number oflaboratory confirmed malaria cases were 22,494 with total slide positivity rate (SPR) of $41.53 \%$. It isin consistence with a study conducted in Kola Diba, Northern Ethiopia, where the total slidepositivity rate was $39.6 \%$ (11). However higher than the findings from Wolayta in South Ethiopia andMetema in Northern Ethiopia where the total slide positivity rate was $33.27 \%$ and $17 \%$ respectively $(15,16)$. In general, the finding indicated that therewasstill malaria burden in thestudy areas, which require due attention to watch the existing intervention strategies and scale it upaccordingly. The prevalence of malaria in the study areas showed fluctuation year-to-year duringthe study period (January 2008-December 2014). The significant rise in malaria prevalence in 2012 as compared to other years in the study area may suggest a history of some low level epidemics attributable to differences in climatic, environmental or human behavioral risk factors in the two years.

From the two health centers considered, the prevalence of malaria cases was higher in Hana Health Center, as the trend indicated that cases increased successively from 2008 to 2012 , though it declined in 2013 and 2014. The variation might be associated with agroecologicaldifferencesof the sites, which fever breedingof mosquitoes and result in relatively more malaria cases in Hana. It might also be due to relative difference in catchment population served in the respective health centers. It could also be due to discrepancy in malaria intervention activities or due to difference in practice of routine documentation and reporting of cases. The sharp decline of the number of malaria cases in 2013 and 2014 could be was, possibly due to strengthening of intervention strategies via distribution of more LLINs, replacing of the old, followed by implementation of indoor residue spray (IRS). Other investigations carried out elsewhere in Ethiopia (17-20) and other African counties such as Zambia (21), Eritrea (22) and Mali (23) also indicated that malaria cases radically fell down after effective intervention strategies using LLINs and IRS with effective insecticides.

In the two study sites,Plasmodiumfalcipariumwas the predominant species (24-28,35). The species was responsible for $61 \%$ of malaria cases followed by $P$. vivax $(32 \%)$. The present result was in agreement with the malaria species distribution in different parts of Ethiopia which indicated that Plasmodium falciparum is the predominant species accounting for greater than $60 \%$ cases, followed by Plasmodium vivax, which is responsible for about $40 \%$ cases $(7,9,13,29)$. The finding was relatively greater than studies done in different parts of Ethiopia $(12,13)$ but less than investigations conducted in South Ethiopia,Wolyta (72\%) (15), North Ethiopia,Koladeba (75\%) (11), Metema (91\%) (16) and the sub-Saharan African countries where $98 \%$ cases were due to $P$. falciparum

DOI: http://dx.doi.org/10.4314/ejhs.v28i3.5 
(27). In both study areas, males were more affected than females which is in agreement with other findings $(11,26,30,31)$. This might be due to prioritizing females in the use of ITNS as of their responsibility to care children, which is in accordance with a previous report (31). Although (25) reported more vulnerability in children of less than 1 year, in our case, adults above 15 years old were the most affected groups like cases reported in other findings $(11,15,32)$. The difference might be associated with exposure difference. Due to the hot weather condition, males have a habit of staying outside their home and sleep under big trees during night and exposed for mosquito bite. Moreover, it might be due to giving more priority to small children in the use of ITN. Such exposure differences to malaria infection was also revealed by other studies $(25,15)$. However, compared to Hana Health Center, infants less than 1 year in Keyafer were more vulnerable to $P$. falciparum;asimilar finding was also reported (30).

As the data of Hana Health Center,the highest peaks of malaria transmission occur in May and June after a heavy rain of March and April and minor in October after moderate rain of August and September. Where asin other part of Ethiopia, the peak malaria transmission follows after a heavy rain of August and September while the minor transmission occurs following the light rain of March and April (33). However, in the case of Keyafer, a high prevalence of malaria transmission was observed during October, which is in accordance with that of the country (33). Metrological variable, particularly rain fall at lag of 1month, were significantly predicted both $P$. falciparum and $P$. vivax incidence in the subsequent monthin Hana Health Center, which is in agreement with other findings (31) in SNNPR and (34) in Amhara Region.

In conclusion, although morbidity and mortality of malaria decreased relatively after introduction of the present controlling strategies, there was still profound burden of malaria in the study sites. $P$. falciparum was the dominant species, and individuals above 15 years and males were more infected. Hence, to reduce the burden of malaria, enhancing the available malaria prevention strategies, early detection and short-term forecasting based on local meteorological factors can be considered.

\section{ACKNOWLEDGMENTS}

The authors would like to thank Hana and KeyaferHealth Center staff and Salamago and Benatsemay district health offices for providing the required data. We acknowledge Hawassa University for sponsoring the study. We would also like to extend our heartiest appreciation to data collectors for offering the essential data from the log book and SNNPR meteorological agency for availing metrological data.

\section{REFERENCES}

1. Dieudonné MM, Thierry LB, Solange EU, et al.Investigation on possible transmission of monkeys' Plasmodium to human in a populations living in the equatorial rainforest of the Democratic republic of Congo. Int J Parasitol Parasites Wildl. 2016; 5:1-4.

2. World Health Organization (WHO). Global and regional disease burden. World malaria day report, 2017.

3. World Health Organization (WHO). Global Malaria Program: World Malaria Report 2014.

4. Murray CJ, Ortblad KF, Guinovart C, et al. 2014. Global, regional, and national incidence and mortality for HIV, tuberculosis, and malaria during 1990-2013: systematic analysis for the Global Burden of Disease Study. Lancet,2013;384:1005-1017.

5. Dawit A, Temesgen Z, Henry GM. Prevalence and risk factors of malaria in Ethiopia. Malar $J$, 2012;11:195-2004.

6. Carter center. Review of malaria control program in Ethiopia and Nigeria. In : Summery proceeding of the 4th annual malaria control program. March 8, 2013. Atlanta, Georgia.

7. Federal Ministry of Health (FMoH). National Malaria Control program Surveillance. In: Summery proceeding. 2012, Addis Ababa, Ethiopia.

8. Federal Ministry of Health $(\mathrm{FMoH})$. National Strategic Plan for Malaria Prevention, Control and Elimination in Ethiopia. 2009, Addis Ababa, Ethiopia.

9. Southern Nations and Nationality Peoples Region (SNNPR). Southern Nations, Nationalities and

DOI: http://dx.doi.org/10.4314/ejhs.v28i3.5 
Peoples' Region Health Bureau Malaria Annual Report, 2013, Hawassa, Ethiopia.

10. Getachew G and Tsige K. Severe Malaria Associated with Plasmodium falciparum and $P$. vivax among Children in Pawe Hospital, Northwest Ethiopia. Malar Res Treat, 2016;5:112.

11. Abebe A, Dagnachew M, Mikire M, Meaza A, Melkamu G. Ten year trend analysis of malaria prevalence in Kola Diba, North Gondar. Parasit Vectors, 2012; 5: 173-183.

12. Wagari D, ChibsaS, Olana D. The distribution and magnitude of malaria in Oromia, Ethiopia. Ethiop $J$ Health Dev, 2004; 18:164-170.

13. National Meteorological Agency, Southern Zone, 2014, Hawassa, Ethiopia.

14. Regional State Bureau of Finance and Economy Development (RFEDO), division of statistics and population. Regional statistical abstract, 2011, Hawassa, Ethiopia.

15. Deresse L, Yusuf H, Solomon A. Trend Analysis of Malaria Occurrence in Wolaita Zone, Southern Ethiopia: Retrospective Cross-Sectional Study. Malar Res Treat, 2015;8:1-9.

16. Getachew F, Abiyu W, Alemtegna G, et al. Prevalence of Malaria from Blood Smears Examination: A Seven-Year Retrospective Study from Metema Hospital, Northwest Ethiopia. Malar Res Treat, 2013; 5:1-6.

17. Legesse Y, Tegegn A, Belachew T. Knowledge, Attitude and Practice about Malaria Transmission and Its Preventive Measures among Households in Urban Areas of Assossa Zone, Western Ethiopia. EthiopJ Health Dev, 2007; 21:157-65.

18. Wagari D, Ahmed A, Yemane B. Review of the interplay between population dynamics and malaria transmission in Ethiopia. EthiopJ Health Dev, 2006; 20:137-144.

19. Kassahun N. "Ethiopia Roll Back Malaria Consultative Mission: Essential Actions to Support the attainment of the Abuja Targets," Ethiopia RBM Country Consultative Mission Final Report, 2004.

20. Aschalew A. and Tadesse D. Current Status of Malaria in Ethiopia: Evaluation of the Burden, Factors for Transmission and Prevention Methods. ActaParasitologicaGlobalis, 2016; 7:01-06.
21. Masaninga F, Chanda E, Chanda-Kapata P, Hamainza B, Masendu HT, Kamuliwo M, Kapelwa W, Chimumbwa J, Govere J, Otten M, Fall IS, Babaniyi O: Review of the malaria epidemiology and trends in Zambia. Asian Pac $J$ Trop Biomed, 2013; 3:89-94.

22. Nyarango PM, Gebremeskel T, Mebrahtu G, Mufunda J, Abdulmumini U, Ogbamariam A, Kosia A, Gebremichael A, Gunawardena D, Ghebrat Y, Okbaldet Y: A steep decline of malaria morbidity and mortality trends in Eritrea between 2000 and 2004: the effect of combination of control methods. Malar J, 2006; 5:33.

23. Rose-Wood ADS, Traore B, Castro MC: Trends in malaria morbidity among health careseeking children under age five in Mopti and Sevare, Mali between 1998 and 2006. Malar J, 2010; 9:319.

24. Ketema T, Getahun K and Bacha K. Therapeutic efficacy of chloroquine against Plasmodium vivax malaria in HalabaKulito town, South Ethiopia. Parasit Vectors, 2011;4:46.

25. WHO. World Malaria Report. Geneva: World Health Organization; 2012.

26. Lelisa S, Wagari D, Ahmed A. Analysis of trends of malaria prevalence in South Western Ethiopia. Malar J, 2014; 13:188.

27. Malaria Indicator Survey, 2011, Ethiopia, Addis Ababa.

28. Lo E, Delenasaw Y, Daibin Z, et al., Molecular epidemiology of Plasmodium vivax and Plasmodium falciparum malaria among Duffypositive and Duffy-negative populations in Ethiopia. Malar J, 2015; 14:84-94.

29. Otten M, Aregawi M, Were W, et al. Initial evidence of reduction of malaria cases and deaths in Rwanda and Ethiopia due to rapid scale-up of malaria prevention and treatment. Malaria $J$, 2009; 8:14.

30. Mengistu H. and Solomon G. Trend analysis of malaria prevalence in ArsiNegelle health center, Southern Ethiopia. J Infect Dis. 2015; 7:1-6.3.

31. Eskindir L. and Lindtjørn B. Model variations in predicting incidence of Plasmodium falciparum malaria using 1998-2007 morbidity and meteorological data from south Ethiopia. Malar $J, 2010$; 9:166-176.

DOI: http://dx.doi.org/10.4314/ejhs.v28i3.5 
32. Kaliyaperumal K and Mammo B. Changes in Malaria Indices in an Ethiopian Health Centre: A Five Year Retrospective Analysis. J Health Scope, 2012; 1:118-126.

33. Ministry of Health. Ethiopian National Malaria Control program. Progress Report 2010, Addis Ababa. Ethiopia.
34. Adisu W. and Belay B. Climate variability and malaria transmission in Fogra district, Ethiopia: 2003 - 2011. SJPH, 2014; 2:234 - 237.

35. Gidie WD. and Dejene HK. Prevalence of malaria and associated factors in BennaTsemay district of pastoralist community, Southern Ethiopia.Trop Dis Travel MedVaccines, 2016; $2: 16$. 